\title{
Prognostic significance of VEGF, VEGF receptors, and microvessel density in diffuse large B cell lymphoma treated with anthracycline-based chemotherapy
}

\author{
Dita Gratzinger ${ }^{1}$, Shuchun Zhao ${ }^{1}$, Robert J Tibshirani ${ }^{2}$, Eric D Hsi ${ }^{3}$, Christine P Hans ${ }^{4}$, Brad Pohlman ${ }^{5}$, Martin Bast ${ }^{6}$, \\ Abraham Avigdor ${ }^{7}$, Ginette Schiby ${ }^{7}$, Arnon Nagler ${ }^{7}$, Gerald E Byrne $\mathrm{Jr}^{8}$, Izidore S Lossos ${ }^{9}$ and Yasodha Natkunam ${ }^{1}$
}

Vascular endothelial growth factor-mediated signaling has at least two potential roles in diffuse large B cell lymphoma: potentiation of angiogenesis, and potentiation of lymphoma cell proliferation and/or survival induced by autocrine vascular endothelial growth factor receptor-mediated signaling. We have recently shown that diffuse large B cell lymphomas expressing high levels of vascular endothelial growth factor protein also express high levels of vascular endothelial growth factor receptor-1 and vascular endothelial growth factor receptor-2. We have now assessed a larger multi-institutional cohort of patients with de novo diffuse large B cell lymphoma treated with anthracycline-based therapy to address whether tumor vascularity, or expression of vascular endothelial growth factor protein and its receptors, contribute to patient outcomes. Our results show that increased tumor vascularity is associated with poor overall survival $(P=0.047)$, and is independent of the international prognostic index. High expression of vascular endothelial growth factor receptor-1 by lymphoma cells by contrast is associated with improved overall survival $(P=0.044)$. The combination of high vascular endothelial growth factor and vascular endothelial growth factor receptor-1 protein expression by lymphoma cells identifies a subgroup of patients with improved overall $(P=0.003)$ and progression-free $(P=0.026)$ survival; these findings are also independent of the international prognostic index. The prognostic significance of overexpression of this ligand-receptor pair suggests that autocrine signaling via vascular endothelial growth factor receptor-1 may represent a survival or proliferation pathway in diffuse large B cell lymphoma. Dependence on autocrine vascular endothelial growth factor receptor-1-mediated signaling may render a subset of diffuse large B-cell lymphomas susceptible to anthracycline-based therapy.

Laboratory Investigation (2008) 88, 38-47; doi:10.1038/labinvest.3700697; published online 12 November 2007

KEYWORDS: angiogenesis; diffuse large B-cell lymphoma; VEGF; VEGF receptor 1; VEGF receptor 2

Vascular endothelial growth factor (VEGF)-mediated angiogenesis has received considerable attention in the context of solid neoplasia, particularly with the clinical use of antiVEGF antibodies and small molecule VEGF receptor (VEGFR) inhibitors. More recently the concept of tumor vascularity has been applied to hematolymphoid neoplasia, with studies quantitating microvessel density in a variety of lymphomas. ${ }^{1}$ In addition to its role in tumor angiogenesis, however, VEGF has an additional potential role in the context of hematolymphoid malignancies: that of an autocrine growth factor, acting on lymphoma cells directly through VEGF receptors. ${ }^{2}$ Indeed, a murine model of human aggressive B cell non-Hodgkin lymphoma xenografts has shown roles for both autocrine VEGFR-1-mediated signaling and for VEGF-mediated angiogenesis in lymphoma. ${ }^{3}$ We recently characterized the immunohistologic expression of VEGF and its receptors VEGFR-1 and VEGFR-2 and quantitated the microvessel density of over 90 diffuse large B-cell

\footnotetext{
${ }^{1}$ Department of Pathology, Stanford University School of Medicine, Stanford, CA, USA; ${ }^{2}$ Department of Health Research and Policy and Statistics, Stanford University, Stanford, CA, USA; ${ }^{3}$ Department of Clinical Pathology, Cleveland Clinic Foundation, Cleveland, OH, USA; ${ }^{4}$ Department of Pathology, University of Nebraska Medical Center, Omaha, NE, USA; ${ }^{5}$ Department of Hematologic Oncology and Blood Disorders, Cleveland Clinic Foundation, Cleveland, OH, USA; ${ }^{6}$ Department of Medicine, University of Nebraska Medical Center, Omaha, NE, USA ${ }^{7}{ }^{7}$ Chaim Sheba Medical Center, Tel Hashomer, Israel; ${ }^{8}$ Department of Pathology, University of Miami, Miami, FL, USA and ${ }^{9}$ Division of Hematology-Oncology and Molecular and Cellular Pharmacology, Department of Medicine, Sylvester Comprehensive Cancer Center, University of Miami, Miami, FL, USA

Correspondence: Dr Y Natkunam, MD, PhD, Department of Pathology, Stanford University School of Medicine, 300 Pasteur Drive, Lane 235, Stanford, CA 94305-5324, USA. E-mail: yaso@stanford.edu
}

Received 28 September 2007; accepted 12 October 2007 
lymphomas. ${ }^{4}$ We found that those lymphomas that express high levels of VEGF protein also express high levels of its receptors VEGFR-1 and VEGFR-2, providing the setting for autocrine signaling via one or both receptors.

In the past decade, numerous factors predictive of outcome in diffuse large B-cell lymphoma have been published and have ranged from single gene products to gene expression signatures encompassing multiple co-ordinately regulated genes; as yet none have been sufficiently validated to come into routine clinical use. ${ }^{5}$ Given the rapidly increasing availability of a variety of pharmaceuticals aimed at the VEGF pathway, the role of angiogenesis and VEGF signaling in diffuse large B-cell lymphoma is of significant interest. In the current study, we addressed whether VEGF-mediated autocrine signaling and/or tumor vascularity is pertinent to patient outcome in a cohort of 182 patients presenting with de novo diffuse large B-cell lymphoma treated with anthracycline-based therapy.

\section{MATERIALS AND METHODS Patient Selection}

Pre-treatment biopsies of 182 patients with de novo diffuse large B-cell lymphoma (DLBCL) treated with an anthracycline-containing chemotherapy (cyclophosphamide, vincristine, doxorubicin, and prednisone (CHOP) or CHOP-like regimens) with clinical follow-up data were used. The biopsy specimens and clinical follow-up data originated from four institutions: Nebraska Medical Center, Cleveland Clinic Foundation, University of Miami, and Chaim-Sheba Medical Center, Israel. Specimens were chosen for this study based on the following criteria: (1) diagnosis of de novo DLBCL; (2) availability of tissue obtained at diagnosis before the initiation of therapy; (3) treatment with an anthracycline-containing chemotherapy (cyclophosphamide, vincristine, adriamycin, and prednisone (CHOP) or CHOP-like regimens); and (4) availability of follow-up and outcome data at the treating institution. All patients were treated with curative intent. Patients who received rituximab together with their chemotherapy or who underwent therapy intensification with stem cell transplantation despite clinical response to initial anthracycline-based regimens were excluded from this study. Institutional Review Board approval was obtained from all participating institutions. In all patients chosen for this study, information was available about staging of the disease by physical examination, bone marrow biopsy, and computed tomography of the chest, abdomen, and pelvis. Patients were staged according to the Ann Arbor system. The following clinical and laboratory data at the time of diagnosis was available: age, gender, performance status, stage, number of extranodal sites involved, levels of serum lactate dehydrogenase and the presence or absence of systemic or ' $\mathrm{B}$ ' symptoms. Based on this information, international prognostic index (IPI) scores could be determined on 174 of the patients. Patients were categorized into either a low-risk group (IPI score 0-2) or a high-risk group (IPI score of 3-5).
None of the patients had a known history of HIV infection or other forms of immunosuppression. Follow-up information was obtained from the patients' medical records and included response to initial therapy based on the Cheson criteria, ${ }^{6}$ progression-free survival and overall survival. Histologic sections were reviewed to confirm the diagnoses. All cases showed a diffuse large cell infiltrate without any evidence of follicles or other low-grade component and were compatible with the histologic features of DLBCL according to the World Health Organization classification of hematopoietic tumors. ${ }^{7}$

\section{Tissue Microarrays}

Standardized methods for tissue fixation (10\% buffered formalin) and processing for paraffin-embedded sections were used at all participating centers. Tissue microarrays of formalin-fixed, paraffin-embedded tissue samples of DLBCL were obtained from Cleveland Clinic Foundation, and the University of Nebraska Medical Center. A TMA of cases from the University of Miami and Chaim-Sheba Medical Center was constructed using a tissue arrayer (Beecher Instruments, Silver Spring, MD, USA), as described previously. ${ }^{8}$ Tissue cores were selected for tissue microarray by characteristic morphology based on examination of hematoxylin and eosin-stained sections, without prior knowledge of immunohistologic stains of individual cases. Two to four representative cores of each case were included to maximize informative cores. Sections of $4-5 \mu$ thickness were cut from the tissue microarrays and placed on glass slides, which were then baked for $1 \mathrm{~h}$ at $60^{\circ} \mathrm{C}$.

\section{Immunohistochemistry and Microvessel Density Counting}

Immunohistochemistry for CD34, VEGFR-1, VEGFR-2, and VEGF was performed as described previously. ${ }^{4}$ For each immunostain $84-88 \%$ of cases were informative, with an average of three cores evaluated per informative case. Stains for VEGFR-1, VEGFR-2, and VEGF were scored as follows: $>30 \%$ of lymphoma cells staining, score $=2 ; 5-30 \%$ of lymphoma cells staining, or weak diffuse staining, score $=1$; and $<5 \%$ of lymphoma cells staining, score $=0$. The highest score obtained from the informative cores was reported. Scores of 2 are denoted as 'high', and scores of 0 or 1 as 'low.' For the combined variable VEGF + R1, the scores for VEGF and VEGFR-1 were added together; combined scores of 3-4 are denoted as 'high', and scores of $0-2$ as 'low'. Microvessel density was quantitated as the number of $\mathrm{CD} 34+$ microvessels (defined as any distinct CD34 + cell or cell cluster), ${ }^{9}$ per high power field at $\times 300(\times 20$ lens, $\times 15$ ocular, field of view $0.7 \mathrm{~mm}$ ) using an Olympus BX45 microscope, with the average score reported. Microvessel density counts denoted 'high' are above the median score of 22.9, and those denoted 'low' are below the median score. Scoring was performed in a blinded manner by DG and checked by YN. Discrepancies (5-10\%) were resolved over a multi-headed microscope. Images of immunohistologic staining were 
acquired using a Nikon Eclipse E400 microscope (Nikon, Tokyo, Japan) and Nikon digital camera (DS-L1; Nikon, Tokyo, Japan), using a $\times 40 / 0.75$ NA Plan Fluor objective lens. Digitized images were processed using Adobe Photoshop 7 image processing and manipulation software (Adobe Systems, San Jose, CA, USA).

\section{Statistical Analysis}

Survival curves were estimated using the product-limit method of Kaplan-Meier and were compared using the logrank test. Multivariate analysis was performed according to the Cox proportional hazards regression model. The $\chi^{2}$ test for independence was used to assess for correlations between categorical variables.

\section{RESULTS}

\section{Patient Characteristics}

The patient characteristics, including prognostically relevant clinical data and IPI, are presented in Table 1 both for the patient group overall and for the patient subgroups scored as low or high for each variable examined. No statistically significant difference in any patient or disease characteristic was present with respect to microvessel density, VEGF, VEGFR-1 or VEGFR-2 status.

\section{Microvessel Density}

In this cohort of patients with de novo DLBCL, microvessel density varies widely (median 22.9 , range, $2-85.5$, Figure $1 \mathrm{a}-\mathrm{c}$ ). Increasing microvessel density is a weak, but statistically significant $(P=0.047)$ predictor of poorer overall survival (Table 2) and is independent of IPI $(P=0.045$, Table 3$)$. Interestingly microvessel density only reaches statistical significance as a predictor of poor overall survival when examined as a linear variable over the whole range of microvessel densities; statistical analysis does not reveal any specific meaningful cutoff. When an arbitrary split is introduced at the median to yield low $v s$ high microvessel density groups, a trend toward poor overall survival remains but does not reach statistical significance $(P=0.065$, Figure $1 d)$. This reflects the fact that while median overall survival differs by over $50 \%$ between the arbitrarily divided low and high microvessel density groups, the $95 \%$ confidence limits overlap broadly (see Table 2$)$. A non-significant $(P=0.17)$ trend is also present toward poor progression-free survival with increasing microvessel density. The predictive power of microvessel density for overall survival remains statistically significant when VEGF, VEGFR-1, and VEGFR-2 expression by lymphoma cells is taken into account $(P=0.036$ for backward stepwise analysis). We found no correlation between increasing microvessel density and lymphoma cell VEGF expression in this patient cohort.

\section{VEGF, VEGFR-1, and VEGFR-2}

High VEGFR-1 expression by lymphoma cells is predictive of improved overall survival $(P=0.044)$; however it does not
Table 1 Patient and disease characteristics: no statistically significant differences present in any category

\begin{tabular}{|c|c|c|c|c|c|c|c|c|c|}
\hline & \multirow[t]{2}{*}{ All } & \multicolumn{2}{|c|}{ MVD } & \multicolumn{2}{|c|}{ VEGF } & \multicolumn{2}{|c|}{ VEGFR-1 } & \multicolumn{2}{|c|}{ VEGFR-2 } \\
\hline & & Low & High & Low & High & Low & High & Low & $\mathrm{H}$ \\
\hline$n$ & 182 & 80 & 80 & 97 & 75 & 97 & 75 & 65 & \\
\hline
\end{tabular}

$\begin{array}{lrrrrrrrrr}\text { Sex } & & & & & & & & \\ \text { Male } & 82 & 40 & 32 & 46 & 31 & 46 & 31 & 24 & 49 \\ \text { Female } & 100 & 40 & 48 & 51 & 44 & 51 & 44 & 41 & 49 \\ & & & & & & & & & \\ \text { Age } & & & & & & & & & \\ <60 & 56 & 22 & 24 & 29 & 23 & 24 & 27 & 21 & 29 \\ \geq 60 & 127 & 58 & 57 & 68 & 52 & 73 & 48 & 44 & 69 \\ \text { Stage } & & & & & & & & & \\ \text { I } & 55 & 28 & 20 & 29 & 24 & 27 & 25 & 19 & 33 \\ \text { II } & 50 & 20 & 24 & 25 & 19 & 28 & 18 & 19 & 22 \\ \text { III } & 30 & 14 & 14 & 15 & 14 & 19 & 11 & 9 & 18 \\ \text { IV } & 47 & 18 & 22 & 28 & 18 & 23 & 21 & 18 & 25\end{array}$

Performance status (ECOG)

$\begin{array}{lrllllllll}0-1 & 152 & 67 & 69 & 80 & 64 & 81 & 65 & 54 & 83 \\ \geq 2 & 30 & 13 & 11 & 17 & 11 & 16 & 10 & 11 & 15\end{array}$

Lactate dehydrogenase

$\begin{array}{llllllllll}\text { Normal } & 97 & 42 & 41 & 50 & 43 & 53 & 38 & 32 & 56 \\ \text { High } & 77 & 35 & 34 & 44 & 27 & 41 & 32 & 31 & 38\end{array}$

\section{Extranodal sites}

$\begin{array}{llllllllll}0-1 & 149 & 62 & 68 & 81 & 59 & 84 & 57 & 54 & 80 \\ \geq 2 & 33 & 18 & 12 & 16 & 16 & 13 & 18 & 11 & 18\end{array}$

International prognostic index ${ }^{\mathrm{a}}$

$\begin{array}{rrrrrrrrrr}0-2 & 122 & 53 & 53 & 63 & 52 & 65 & 51 & 42 & 69 \\ 3-5 & 52 & 25 & 22 & 31 & 19 & 29 & 20 & 21 & 26\end{array}$

B symptoms ${ }^{\mathrm{a}}$

$\begin{array}{lrllllllll}\text { Absent } & 127 & 62 & 50 & 66 & 54 & 63 & 57 & 44 & 70 \\ \text { Present } & 42 & 14 & 22 & 22 & 17 & 25 & 14 & 18 & 22\end{array}$

${ }^{\mathrm{a}}$ Data was missing in these categories for some patients.

reach statistical significance as a predictor of overall survival in multivariate analysis as compared with the IPI $(P=0.064)$. Patients with high lymphoma cell VEGFR-1 expression also show a trend $(P=0.054)$ toward improved progression-free 

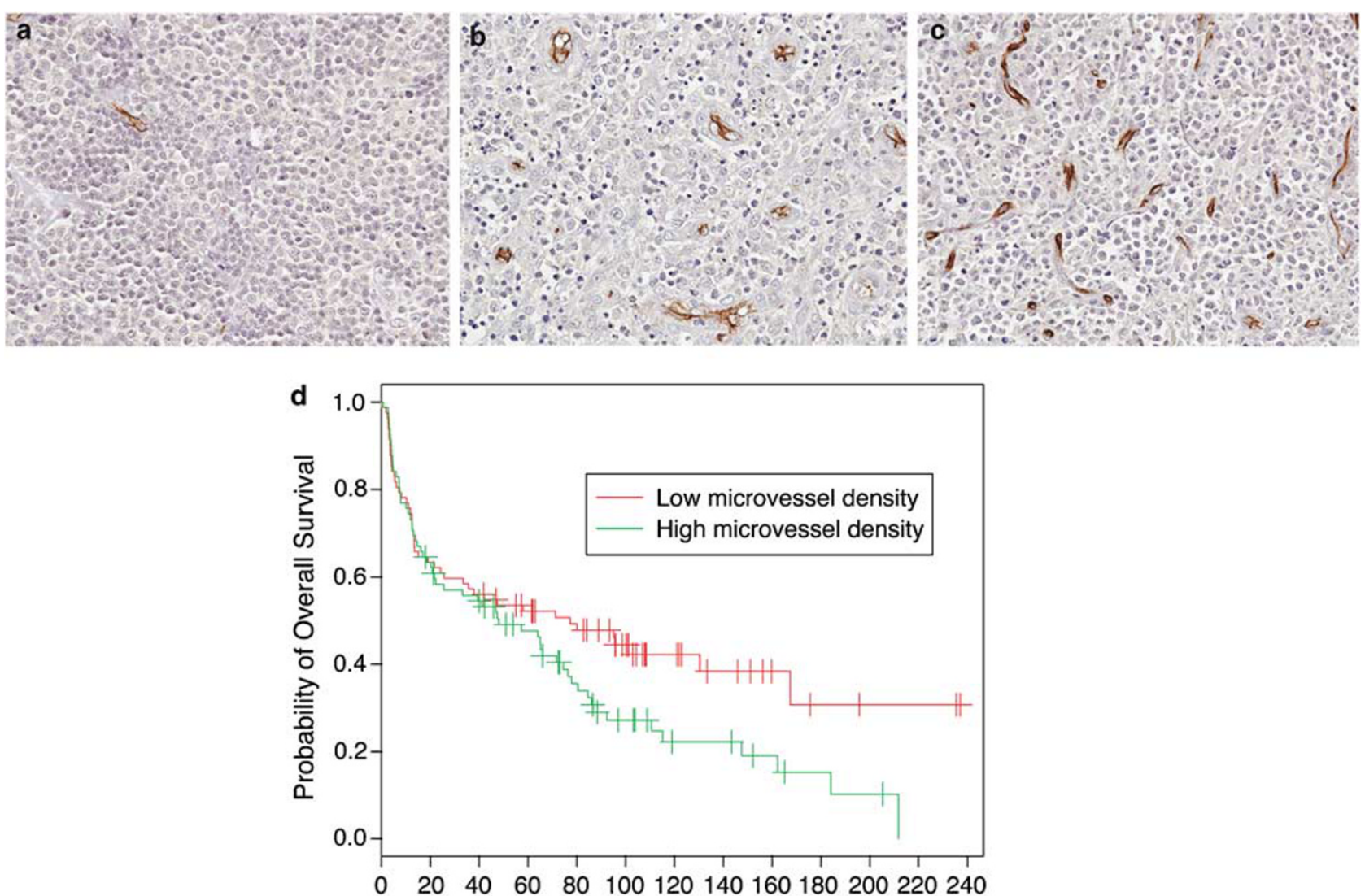

Number at risk:

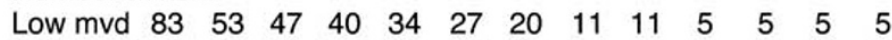

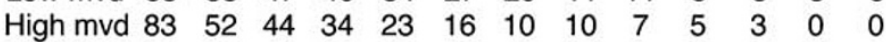

Figure 1 Increased microvessel density is associated with poor overall survival in patients with DLBCL. The range of microvessel density in DLBCL is broad: (a) low microvessel density, (b) median microvessel density, (c) high microvessel density (anti-CD34, $400 \times$ ). (d) Kaplan-Meier analysis shows poor overall survival with increasing microvessel density $(P=0.047)$.

Table 2 Increasing microvessel density is associated with poorer overall survival

\begin{tabular}{|c|c|c|c|c|}
\hline & \multicolumn{2}{|c|}{ Overall survival } & \multicolumn{2}{|c|}{ Progression-free survival } \\
\hline & Low & High & Low & High \\
\hline Median (months) & 77 & 48 & 47 & 28 \\
\hline 95\% confidence limits & 33 to $\infty^{a}$ & 22 to 78 & 16 to 71 & 10 to 57 \\
\hline Linear $P$-value ${ }^{\mathrm{b}}$ & \multicolumn{2}{|c|}{0.047} & \multicolumn{2}{|c|}{0.27} \\
\hline Categorical $P$-value $\mathrm{b}^{\mathrm{b}}$ & \multicolumn{2}{|c|}{0.065} & \multicolumn{2}{|c|}{0.17} \\
\hline
\end{tabular}

\footnotetext{
${ }^{\mathrm{a}}$ The infinity symbol $(\infty)$ indicates that the upper confidence limit is out of range.

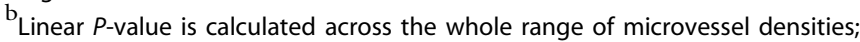
categorical $P$-value compares low vs high microvessel density groups. Italicized $P$-values are statistically significant $(P<0.05)$.
}

survival, which does not reach statistical significance (Table 4, Figure 2). A weaker trend is present for improved overall survival with high lymphoma cell VEGF expression
Table 3 Microvessel density is independent of IPI and VEGF, VEGFR-1 and VEGFR-2 as a predictor of poorer overall survival on multivariate analysis

\begin{tabular}{cccc}
\hline \multicolumn{2}{c}{ IPI } & VEGF, VEGFR-1, VEGFR-2 (backward stepwise analysis) \\
\hline$Z$-score & $P$-value & $Z$-score & $P$-value \\
\hline 2.00 & 0.045 & 2.10 & 0.036 \\
\hline
\end{tabular}

Italicized $P$-values are statistically significant $(P<0.05)$.

$(P=0.089)$ and, to a lesser extent, with high lymphoma cell VEGFR-2 expression (Table 4). In the case of both VEGFR-1 and VEGF, the median overall survival in the high expression group is about twice that in the low expression group (86 vs 39 months for VEGFR-1 and 80 vs 41 months for VEGF, respectively). It is worth emphasizing that in all cases, strong expression of VEGF or its receptors by lymphoma cells is associated with improved overall survival, or with a trend toward improved overall survival. 


\section{VEGF and VEGFR-1 in Combination}

In our previous work ${ }^{4}$ we noted a strong statistically significant association among the expression of VEGF, VEGFR1 , and VEGFR-2 by lymphoma cells. In the current cohort of cases we again note a high degree of concordance among lymphoma specimens that show high expression levels of VEGF, VEGFR-1, and VEGFR-2 (Figure 3, Table 5). This

Table 4 High VEGFR-1 is associated with better overall survival and a trend toward better progression-free survival

\begin{tabular}{|c|c|c|c|c|}
\hline & \multicolumn{2}{|c|}{ Overall survival } & \multicolumn{2}{|c|}{ Progression-free survival } \\
\hline & Low & High & Low & High \\
\hline \multicolumn{5}{|l|}{ VEGFR-1 } \\
\hline Median (months) & 39 & 86 & 24 & 53 \\
\hline 95\% confidence limits & $20-76$ & $57-171$ & $12-49$ & $36-92$ \\
\hline$P$-value & \multicolumn{2}{|c|}{0.044} & \multicolumn{2}{|c|}{0.054} \\
\hline \multicolumn{5}{|l|}{ VEGF } \\
\hline Median (months) & 41 & 80 & 28 & 53 \\
\hline 95\% confidence limits & $22-78$ & $57-171$ & $12-48$ & $38-89$ \\
\hline$P$-value & \multicolumn{2}{|c|}{0.089} & \multicolumn{2}{|c|}{0.14} \\
\hline \multicolumn{5}{|l|}{ VEGFR-2 } \\
\hline Median (months) & 44 & 66 & 24 & 49 \\
\hline 95\% confidence limits & $19-88$ & $47-111$ & $12-53$ & $28-72$ \\
\hline$P$-value & \multicolumn{2}{|c|}{0.23} & \multicolumn{2}{|c|}{0.10} \\
\hline
\end{tabular}

Italicized $P$-values are statistically significant $(P<0.05)$.

a

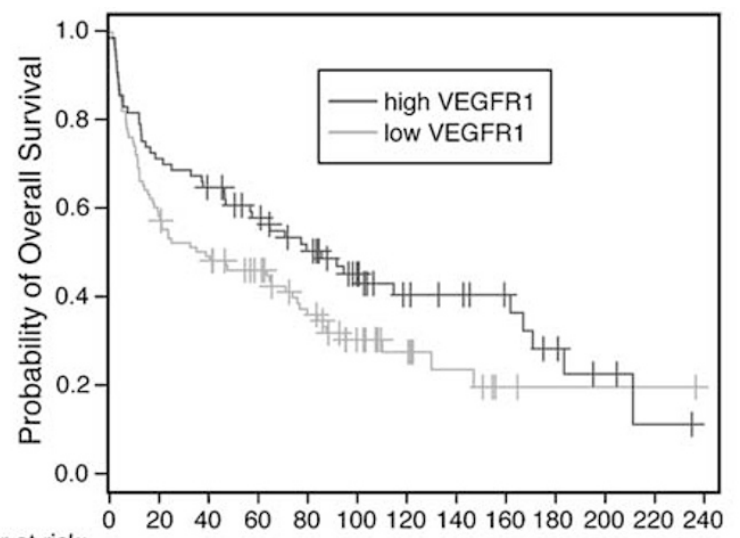

Number at risk:

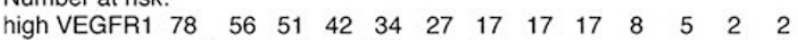

$\begin{array}{lllllllllllllll}\text { low VEGFR1 } & 102 & 61 & 50 & 44 & 30 & 21 & 11 & 7 & 6 & 6 & 6 & 6 & 6\end{array}$ together with the concordant trend towards improved survival with higher expression of VEGF, VEGFR-1, and VEGFR-2 by lymphoma cells, suggested to us a possible interaction between VEGF and one or more of its receptors in predicting survival. Since VEGFR-1 and VEGF were the strongest predictors of survival, we assessed whether patients whose lymphoma specimens show concomitant high expression of both VEGF and VEGFR-1 represent a particularly prognostically favorable group (Table 6).

The new variable, denoted 'VEGF + R1', represents the sum of the values assigned for VEGF and VEGFR-1 staining (weighted equally), where 0 is no staining, 1 is partial $(<30 \%)$, and 2 is positive staining $(>30 \%)$. VEGF $+\mathrm{R} 1$ is predictive of both overall $(P=0.003)$ and progression-free survival $(P=0.026)$ when calculated across the range of values of VEGF + R1 (0-4). Moreover VEGF + R1 remains an independent predictor of both overall survival $(P=0.0055)$ and progression-free survival $(P=0.021)$ in multivariate analysis that includes the IPI (Table 7$)$. When VEGF + R1 scores are evaluated only as low $(0-2) v s$ high (3-4) and not across the whole range of values (0-4), patients with a high VEGF + R1 score (100 patients) show improved overall $(P=0.002)$ and a trend toward improved progression-free $(P=0.058)$ survival compared with patients with a low VEGF + R1 score (75 patients) (Figure 4). Addition of VEGFR-2 to the model (summing up the scores for VEGF, VEGFR-1, and VEGFR-2) does not add any further predictive value (data not shown).

\section{DISCUSSION}

Colorectal carcinoma serves as the prototypical example of a solid tumor where high microvessel density portends poor outcome, providing the rationale for successful use of anti-VEGF therapy in metastatic colorectal carcinoma. ${ }^{10}$ In the setting of hematolymphoid neoplasia high microvessel

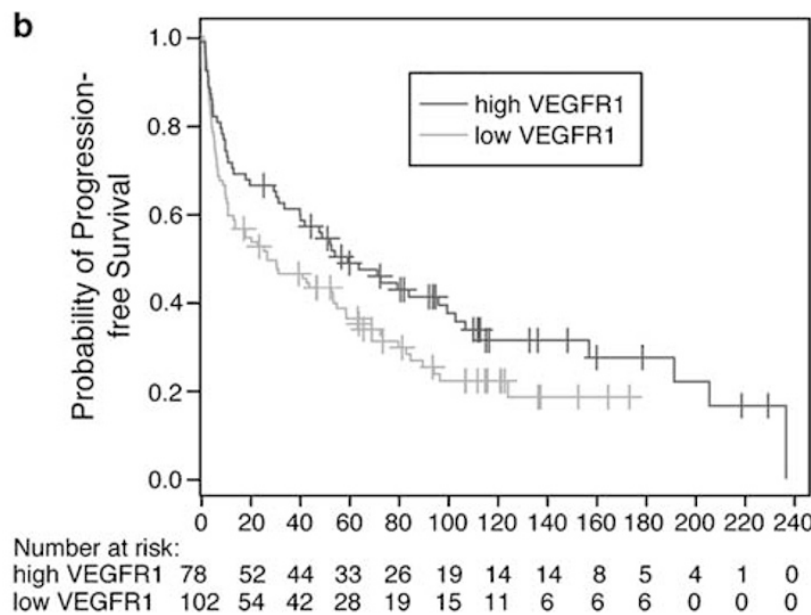

Figure 2 High VEGFR-1 predicts improved overall survival. (a) Kaplan-Meier analysis shows significantly improved overall survival in the high VEGFR-1 as compared to the low VEGFR-1 subgroup $(P=0.044)$. (b) A trend is also present toward improved progression-free survival in the high VEGFR-1 group $(P=0.054)$. 

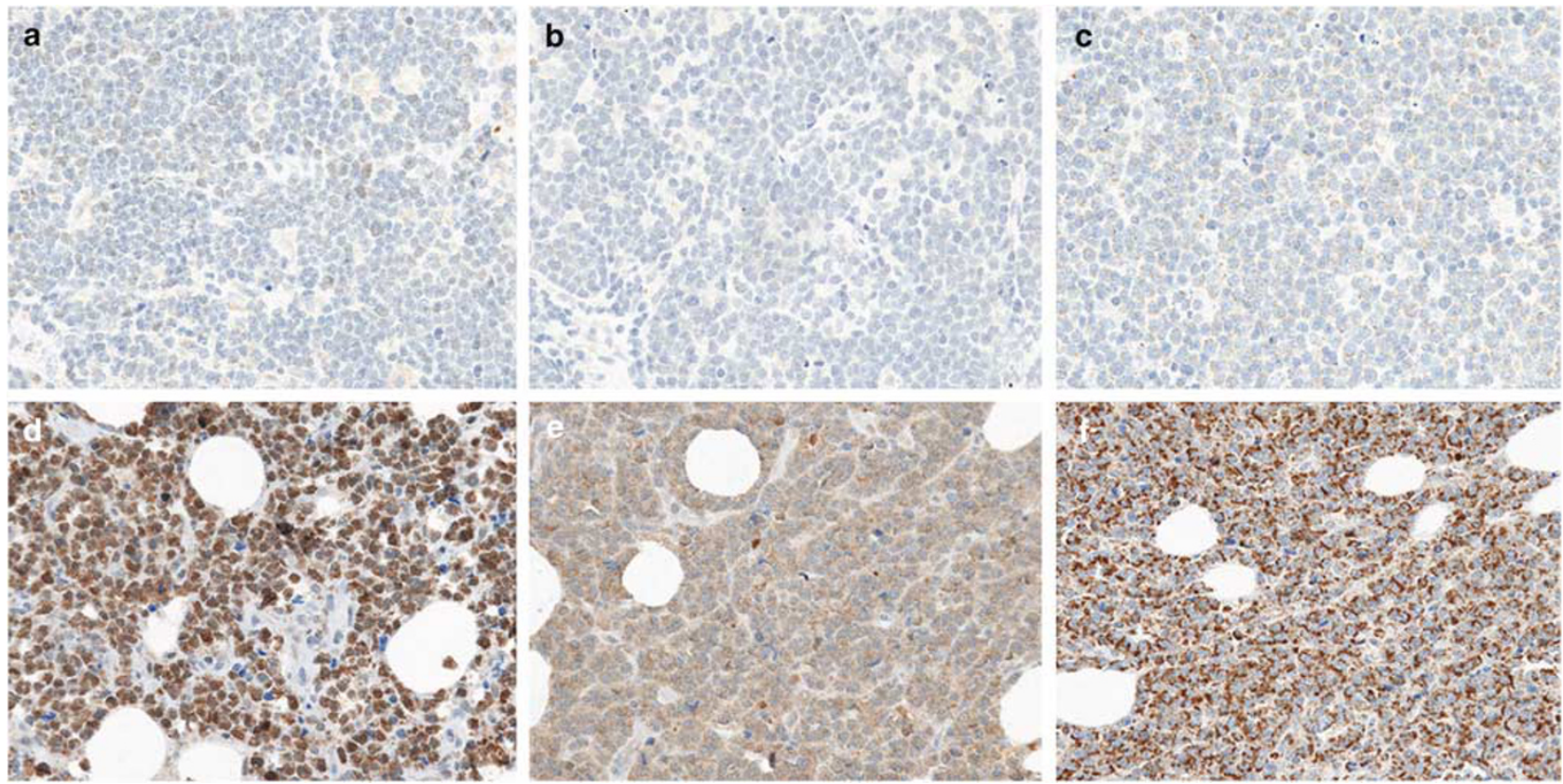

Figure 3 VEGF expression correlates with expression of its receptors VEGFR-1 and VEGFR-2. (a-c) DLBCL with low expression of VEGF, VEGFR-1, and VEGFR-2. (b-d) DLBCL with high expression of VEGF, VEGFR-1, and VEGFR-2 (a, d anti-VEGF; b, e anti-VEGFR-1; c, $\mathbf{f}$ anti-VEGFR-2; $400 \times$ ).

Table 5 Expression of VEGF, VEGFR-1, and VEGFR-2 covaries

VEGFR-1

VEGFR-2

\begin{tabular}{|c|c|c|}
\hline \multicolumn{3}{|l|}{ VEGF } \\
\hline$P$-value & 0.00025 & $3.07 e-06$ \\
\hline$\chi$ value & 21.54 & 31.21 \\
\hline \multicolumn{3}{|l|}{ VEGFR-1 } \\
\hline$P$-value & & 0.019 \\
\hline$\chi$ value & & 11.79 \\
\hline
\end{tabular}

Italicized $P$-values are statistically significant $(P<0.05)$.

density predicts progression ${ }^{11}$ and poorer overall survival in multiple myeloma ${ }^{12}$ but is a favorable prognostic factor in follicular lymphoma. ${ }^{13}$ A single study addressing the relationship of vascularity to chemosensitivity in DLBCL ${ }^{14}$ found no correlation with microvessel density, but was limited by small sample size ( 36 patients) and potentially by the use of factor VIII and CD31, instead of the more sensitive tumor vessel marker CD34, to assess microvessel density. ${ }^{15}$

In the current study, we found that increasing microvessel density is a poor prognostic indicator for overall but not progression-free survival, and is independent of the IPI. Microvessel density is statistically significant only when computed as a continuous variable over the whole range of vascularity. A specific cut-point that would risk-stratify
Table 6 The combination of high VEGFR-1 and VEGF (denoted VEGF+R1) is a stronger predictor of better overall and progression-free survival

\begin{tabular}{|c|c|c|c|c|}
\hline & \multicolumn{2}{|c|}{ Overall survival } & \multicolumn{2}{|c|}{ Progression-free survival } \\
\hline & Low & High & Low & High \\
\hline Median (months) & 29 & 92 & 22 & 53 \\
\hline 95\% confidence limits & $18-72$ & $65-171$ & $10-48$ & $38-76$ \\
\hline Linear $P$-value ${ }^{a}$ & \multicolumn{2}{|c|}{0.003} & \multicolumn{2}{|c|}{0.026} \\
\hline Categorical $P$-value ${ }^{a}$ & \multicolumn{2}{|c|}{0.002} & \multicolumn{2}{|c|}{0.058} \\
\hline
\end{tabular}

\footnotetext{
a Linear $P$-value is calculated across the whole range of values of VEGF+R1; categorical $P$-value compares low vs high VEGF+R1 groups.

Italicized $P$-values are statistically significant $(P<0.05)$.
}

Table 7 VEGF+R1 is independent of IPI for both overall and progression-free survival on multivariate analysis

\begin{tabular}{lclcc}
\hline \multicolumn{2}{c}{ Overall survival } & & \multicolumn{2}{c}{ Progression-free survival } \\
\cline { 1 - 1 } -score & $P$-value & & $Z$-score & $P$-value \\
\hline-2.78 & 0.0055 & & -2.31 & 0.021 \\
\hline
\end{tabular}

Italicized $P$-values are statistically significant $(P<0.05)$.

patient populations with statistical significance is lacking. Although increased vascularity may be associated with clinical aggressiveness in DLBCL, this association could also 
a

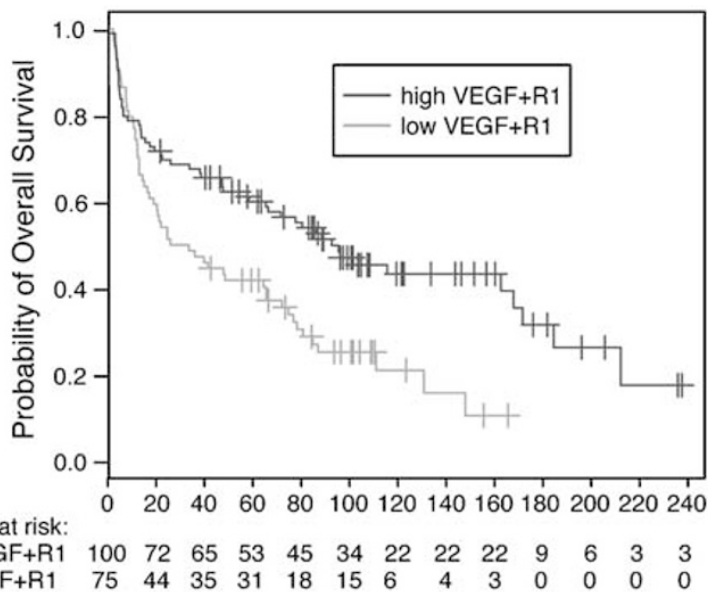

b

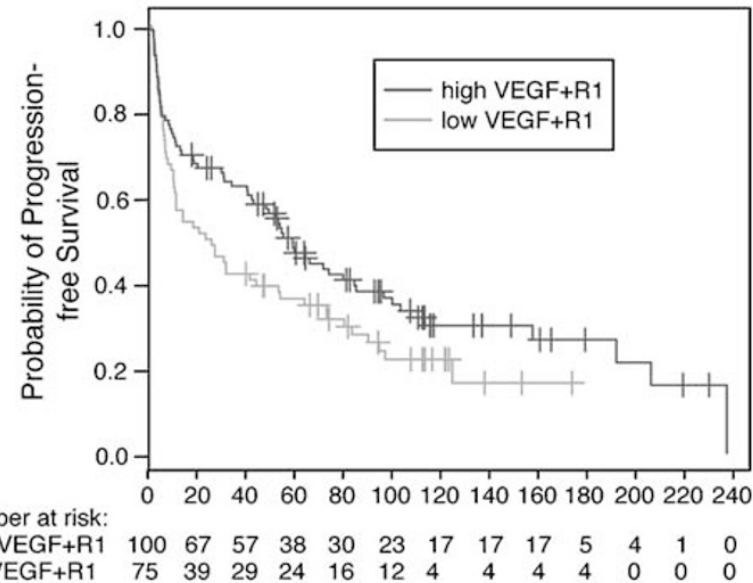

Figure 4 The combination of high VEGF and high VEGFR-1 correlates with improved overall and progression-free survival. VEGF + R1 is the combined score of the individual scores for VEGF and VEGFR-1, which are weighted equally. (a) Kaplan-Meier analysis shows improved overall with increasing VEGF $+\mathrm{R} 1$ score $(P=0.003)$. (b) While Kaplan-Meier analysis also shows increased progression-free survival with increasing VEGF $+\mathrm{R} 1$ score $(P=0.026)$.

represent an epiphenomenon reflecting high circulating levels of angiogenic growth factors ${ }^{16}$ and inflammatory cytokines ${ }^{17}$ which identify a patient population at risk for poor outcome. In fact, in a small (13 patient) safety study of the anti-VEGF antibody bevacizumab in addition to anthracycline-based chemotherapy and rituximab, there was no correlation between baseline microvessel density and outcome. ${ }^{18}$

In a previous study assessing the relationships among microvessel density and expression of VEGF and its receptors in $\mathrm{DLBCL}^{4}$ we found a strong positive association among the expression of VEGF and its receptors VEGFR-1 and VEGFR-2 (indicating a possible autocrine signaling role for VEGF in lymphomagenesis) as well as a positive association between microvessel density and local expression of VEGF by lymphoma cells (suggesting a possible paracrine signaling role for VEGF in local angiogenesis). While the strong positive association among expression of VEGF and its receptors was confirmed, we found no correlation between VEGF expression and local vascularity in the current study. This discrepancy may be due to the influence of local and systemic angiogenic factors: the presence of alternate, locally produced angiogenic growth factors such as basic fibroblast growth factor could potentially influence local angiogenesis; the expression patterns of VEGF and its receptors may effect the availability of VEGF to the local vasculature; and circulating angiogenic factor levels may overwhelm local paracrine angiogenic VEGF function. In comparison to our prior study, fewer lymphomas in the current study were found to express high VEGF levels (42 vs 60\%, $P<0.01, \chi 13.7)$ and a larger number were found to express high VEGFR-1 (43 vs 30\%, $P<0.05, \chi 10.0)$ and high VEGFR-2 (60 vs $44 \%, P<0.02$, $\chi$ 12.0). Transgenic mouse models have shown that during vasculogenesis VEGFR-1 acts by depleting the locally produced VEGF that is available to adjacent nascent vasculature. ${ }^{19}$ This depletion of VEGF is likely to be enhanced in lymphomas with higher VEGF receptor as compared to VEGF expression, complicating the relationship between VEGF expression by the lymphoma and microvessel density.

In univariate analysis we found that high expression of VEGFR-1 by lymphoma cells is predictive of improved overall survival, an effect that is independent of IPI. In a human DLBCL xenograft model in mice, ${ }^{3}$ blockade of human VEGFR-1 (but not VEGFR-2) slowed tumor growth, suggesting an autocrine role for VEGFR-1 in lymphomagenesis. If indeed VEGFR-1 plays such a role in the clinical course of human DLBCL, one would expect tumors with high VEGFR-1 expression to also express high levels of its ligand VEGF. Indeed, the correlation between expression of VEGF and VEGFR-1 (and VEGFR-2) by lymphoma cells was highly statistically significant both in our previous study ${ }^{4}$ and in the current study. Furthermore, if VEGF and VEGFR-1 are involved in an autocrine signaling pathway, one would expect that high VEGF expression would have the same relationship to prognosis as high VEGFR-1 expression. Indeed, while only VEGFR-1 reaches statistical significance as a prognostic marker for overall survival in our cohort of cases, there remains a trend for high expression levels of both VEGF and VEGFR-1 to be associated with improved overall survival.

It is important to distinguish between VEGF expression by the lymphoma as assessed by immunohistochemistry, and circulating levels of VEGF measured in serum samples. Patients with poor prognosis DLBCLs have high serum levels of VEGF ${ }^{16}$ as well as a variety of other growth factors ${ }^{16,20}$ and inflammatory cytokines. ${ }^{1721}$ Expression of VEGF and its receptor VEGFR-1, by contrast, is associated with good prognosis in this analysis. The relationship, if any, between serum levels of VEGF and local expression of VEGF by the lymphoma is unknown.

We have noted that VEGF and its receptors are expressed at high levels in a subset of DLBCL, and that the combination 
of high VEGF and high VEGFR-1 expression by lymphoma cells (denoted VEGF + R1) is predictive of both overall and progression-free survival independent of the IPI. Incorporating VEGFR-2 status does not improve prognostic value with regard to survival. This raises the question of autocrine signaling via a self-contained VEGF-VEGFR-1 pathway in a subset of DLBCLs (Figure 5a). The plausibility of this model is bolstered by the previously mentioned study of human DLBCL xenografts in mice, which supports an autocrine role for VEGFR-1 but not VEGFR-2 in lymphomagenesis. ${ }^{3}$ Despite their similar general structures and overlapping distributions and functions, signaling via the VEGFR-1 and VEGFR-2 receptor tyrosine kinases is quite distinct, involving different intracytoplasmic signaling pathways. $^{22,23}$

In hematopoietic stem cells VEGF signaling via a 'private' autocrine loop not accessible to extracellular manipulation has been suggested. ${ }^{24}$ In particular, survival and proliferation were inhibited by intracellularly acting small molecule inhibitors of VEGF signaling but not to extracellular depletion of VEGF by recombinant soluble VEGFR-1-immunoglobulin-G chimeric protein. This may have implications for any similar autocrine loop present in DLBCL, which may potentially be more susceptible to inhibition by small molecule tyrosine kinase inhibitors than larger recombinant proteins aimed at depleting VEGF (Figure $5 b$ ).

We have suggested that a VEGF-VEGFR-1 autocrine loop may be active in a subset of DLBCLs. Why then would a patient group with high expression of both the ligand and its receptor show better overall and progression-free survival? One hypothesis is that those lymphomas which are dependent on this autocrine loop for proliferation and/or survival are more susceptible to standard anthracycline-based chemotherapy, perhaps through effects directly on VEGF and/or VEGFR-1 or on downstream signaling pathways. As lymphoma is not generally sampled post-treatment except in the case of treatment failure, there is no published data on the effect of standard chemotherapeutic regimens on VEGF and VEGFR-1 expression in lymphoma. Serum VEGF levels are at best an imperfect marker for VEGF levels within the lymphoma itself but are readily obtained; in a study of 64 patients with aggressive non-Hodgkin's lymphoma (61\% DLBCL), early response over the first 3 weeks of treatment correlated with drops in serum VEGF (and IL-6). ${ }^{25}$

Although no data exist on the effect of CHOP (cyclophosphamide, doxorubicin, vincristine, and prednisone) on VEGF or its receptors in DLBCL, evidence for downregulation of VEGF mRNA expression by these agents exists in several other model systems. ${ }^{26-31}$ A model of the potential impact of anthracycline (doxorubicin) administration is presented in Figure 5c. In a DLBCL dependent on autocrine VEGF-mediated signaling for proliferation and/or survival, chemotherapy-mediated abrogation of local VEGF mRNA expression might slow growth or even predispose to apoptosis.

The usual rationale of anti-VEGF therapy is to decrease angiogenesis and 'normalize' immature leaky tumor vasculature, although a role for disruption of autocrine VEGF
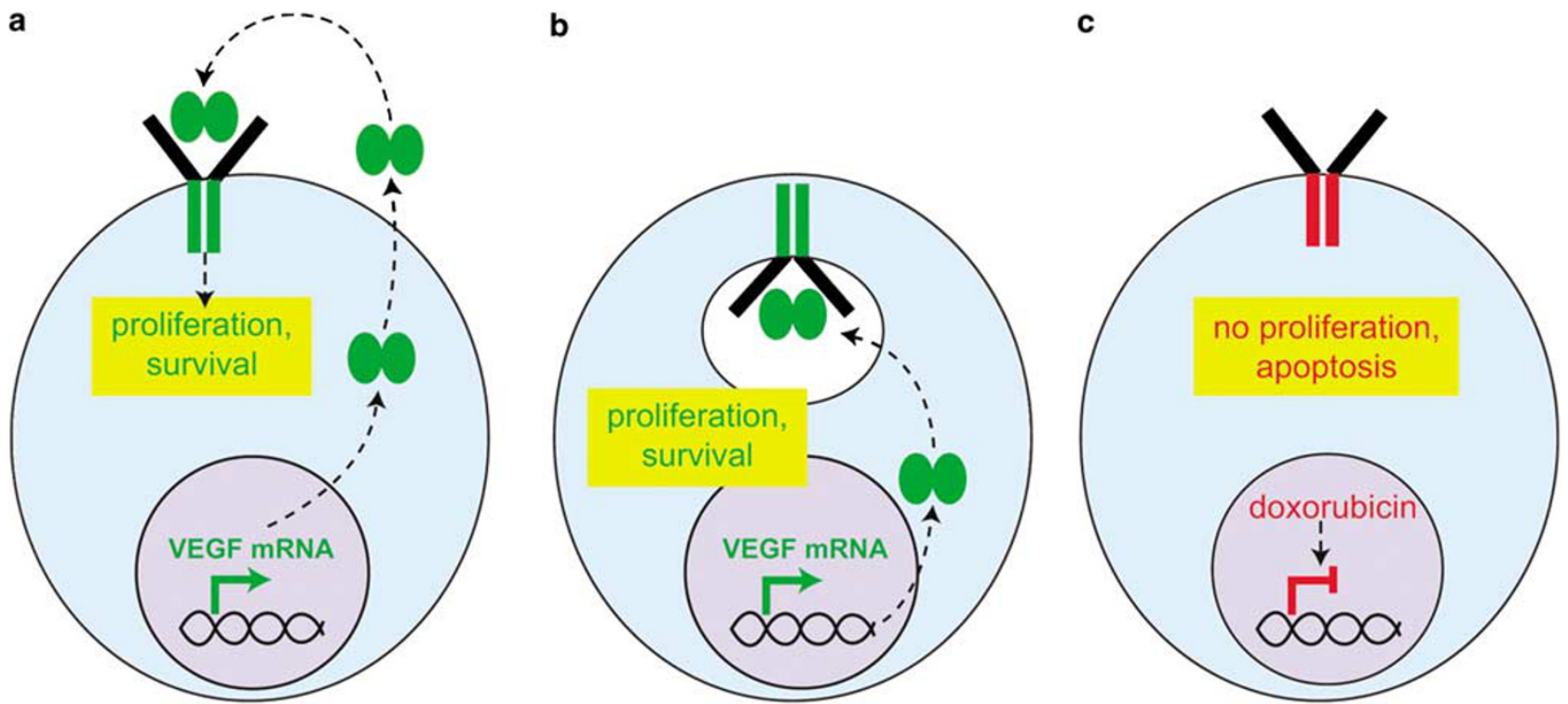

Figure 5 DLBCL coexpressing high levels of VEGF and VEGFR-1 may be dependent on autocrine signaling for survival or proliferation. A hypothetical model of a lymphoma cell expressing high levels of VEGF and VEGFR-1. (a) High levels of VEGF mRNA are transcribed and VEGF protein is secreted locally, binds cell-surface VEGFR-1, and initiates intracellular survival and/or proliferation signaling cascades. (b) The autocrine feedback loop may be 'private,' and thus may be accessible to cell permeable VEGFR-1 tyrosine kinase inhibitors but not recombinant proteins that act as extracellular VEGF sinks. (c) Components of standard anthracycline-based chemotherapy such as doxorubicin may interrupt this autocrine feedback loop, perhaps by inhibiting transcription of VEGF mRNA, leading to decreased proliferation signals and/or increased apoptosis. 
receptor-mediated signaling has also been postulated. ${ }^{2,32}$ Our data shows that expression of VEGF together with VEGFR-1 is prognostically important, supporting a role for autocrine VEGFR-1-mediated signaling in the clinical course of DLBCL. Although further validation in an independent, preferably prospective study is necessary to verify the prognostic significance of expression of VEGFR-1 in combination with VEGF, the fact that two independent studies confirm the strong association between expression of VEGF and VEGFR1 in DLBCL suggests that the biology is real.

Inhibitors directed at both VEGF and its receptors, including antibodies, recombinant proteins and orally active small molecules, are in various stages of testing in both solid tumors and hematopoietic malignancies. The serious vascular side effects of VEGF-directed therapy ${ }^{33}$ (stroke, myocardial infarction) is of particular concern in the substantial proportion of DLBCL patients over the age of 65 and the relative side effect profiles of the numerous VEGF receptor inhibitors will be an important consideration in clinical practice. As clinical testing of VEGF-directed therapy in DLBCL continues, the efficacy of the various available inhibitors in disrupting autocrine VEGFR-1-mediated signaling should be considered and the relationship of VEGF and VEGFR-1 expression at baseline to therapy response should be explored. It will also be interesting to see whether the prognostic impact of VEGF and VEGFR-1 expression remains relevant in patients treated with rituximab in addition to anthracycline-based chemotherapy. However, regardless of the outcome of such a study, the results presented here remain relevant given their implications for another therapeutic target currently moving into clinical practice, namely VEGF and VEGF-receptor signaling.

\section{ACKNOWLEDGEMENT}

This work was supported by NIH CA34233, NIH CA109335 and the Dwoskin Family Foundation.

\section{DISCLOSURE/DUALITY OF INTEREST}

The authors declare no competing financial interest.

1. Koster A, Raemaekers JM. Angiogenesis in malignant lymphoma. Curr Opin Oncol 2005;17:611-616.

2. Podar K, Anderson KC. The pathophysiologic role of VEGF in hematologic malignancies: therapeutic implications. Blood 2005;105:1383-1395.

3. Wang ES, Teruya-Feldstein J, Wu $\mathrm{Y}$, et al. Targeting autocrine and paracrine VEGF receptor pathways inhibits human lymphoma xenografts in vivo. Blood 2004;104:2893-2902.

4. Gratzinger D, Zhao S, Marinelli RJ, et al. Microvessel density and expression of vascular endothelial growth factor and its receptors in diffuse large B-cell lymphoma subtypes. Am J Pathol 2007;170: 1362-1369.

5. Lossos IS, Morgensztern D. Prognostic biomarkers in diffuse large B-cell lymphoma. J Clin Oncol 2006;24:995-1007.

6. Cheson BD, Horning SJ, Coiffier B, et al. Report of an international workshop to standardize response criteria for non-Hodgkin's lymphomas. NCI Sponsored International Working Group. J Clin Oncol 1999;17:1244.

7. Jaffe ES, Harris NL, Stein $\mathrm{H}$, et al. Pathology and Genetics of Tumors of the Hematopoietic and Lymphoid Tissues. IARC Press: Lyon, 2001.
8. Natkunam Y, Lossos IS, Taidi B, et al. Expression of the human germinal center-associated lymphoma (HGAL) protein, a new marker of germinal center B-cell derivation. Blood 2005;105:3979-3986.

9. Weidner N, Semple JP, Welch WR, et al. Tumor angiogenesis and metastasis-correlation in invasive breast carcinoma. N Engl J Med 1991:324:1-8.

10. Giatromanolaki A, Sivridis E, Koukourakis MI. Angiogenesis in colorectal cancer: prognostic and therapeutic implications. Am J Clin Oncol 2006;29:408-417.

11. Vacca $A$, Ribatti $D$, Roncali $L$, et al. Bone marrow angiogenesis and progression in multiple myeloma. Br J Haematol 1994;87:503-508.

12. Kumar $S$, Gertz MA, Dispenzieri A, et al. Prognostic value of bone marrow angiogenesis in patients with multiple myeloma undergoing high-dose therapy. Bone Marrow Transplant 2004;34: 235-239.

13. Koster A, van Krieken JH, Mackenzie MA, et al. Increased vascularization predicts favorable outcome in follicular lymphoma. Clin Cancer Res 2005;11:154-161.

14. Bairey O, Zimra Y, Kaganovsky E, et al. Microvessel density in chemosensitive and chemoresistant diffuse large B-cell lymphomas. Med Oncol 2000;17:314-318.

15. Norrby K, Ridell B. Tumour-type-specific capillary endothelial cell stainability in malignant B-cell lymphomas using antibodies against CD31, CD34 and Factor VIII. Apmis 2003;111:483-489.

16. Salven P, Orpana A, Teerenhovi L, et al. Simultaneous elevation in the serum concentrations of the angiogenic growth factors VEGF and bFGF is an independent predictor of poor prognosis in non-Hodgkin lymphoma: a single-institution study of 200 patients. Blood 2000;96:3712-3718.

17. Seymour JF, Talpaz M, Cabanillas F, et al. Serum interleukin-6 levels correlate with prognosis in diffuse large-cell lymphoma. J Clin Oncol 1995; 13:575-582.

18. Ganjoo KN, An CS, Robertson MJ, et al. Rituximab, bevacizumab and CHOP (RA-CHOP) in untreated diffuse large B-cell lymphoma: safety, biomarker and pharmacokinetic analysis. Leuk Lymphoma 2006;47:998-1005.

19. Hiratsuka S, Minowa O, Kuno J, et al. Flt-1 lacking the tyrosine kinase domain is sufficient for normal development and angiogenesis in mice. Proc Natl Acad Sci USA 1998;95:9349-9354.

20. Bono $\mathrm{P}$, Teerenhovi $\mathrm{L}$, Joensuu $\mathrm{H}$. Elevated serum endostatin is associated with poor outcome in patients with non-Hodgkin's lymphoma. Cancer 2003;97:2767-2775.

21. Blay JY, Burdin N, Rousset $F$, et al. Serum interleukin-10 in non-Hodgkin's lymphoma: a prognostic factor. Blood 1993;82: 2169-2174.

22. Cross MJ, Dixelius J, Matsumoto T, et al. VEGF-receptor signal transduction. Trends Biochem Sci 2003;28:488-494.

23. Olsson AK, Dimberg A, Kreuger J, et al. VEGF receptor signaling-in control of vascular function. Nat Rev Mol Cell Biol 2006;7:359-371.

24. Gerber HP, Malik AK, Solar GP, et al. VEGF regulates haematopoietic stem cell survival by an internal autocrine loop mechanism. Nature 2002;417:954-958.

25. Pedersen LM, Klausen TW, Davidsen UH, et al. Early changes in serum IL- 6 and VEGF levels predict clinical outcome following first-line therapy in aggressive non-Hodgkin's lymphoma. Ann Hematol 2005;84:510-516.

26. Duyndam MC, van Berkel MP, Dorsman JC, et al. Cisplatin and doxorubicin repress vascular endothelial growth factor expression and differentially down-regulate Hypoxia-inducible Factor I activity in human ovarian cancer cells. Biochem Pharmacol 2007:74:191-201.

27. Volm M, Koomagi R, Mattern J, et al. Angiogenic growth factors and their receptors in non-small cell lung carcinomas and their relationships to drug response in vitro. Anticancer Res 1997;17: 99-103.

28. Bottini A, Generali D, Brizzi MP, et al. Randomized phase II trial of letrozole and letrozole plus low-dose metronomic oral cyclophosphamide as primary systemic treatment in elderly breast cancer patients. J Clin Oncol 2006;24:3623-3628.

29. Machein MR, Kullmer J, Ronicke V, et al. Differential downregulation of vascular endothelial growth factor by dexamethasone in normoxic and hypoxic rat glioma cells. Neuropathol Appl Neurobiol 1999;25: 104-112. 
30. Iwai A, Fujii $\mathrm{Y}$, Kawakami $\mathrm{S}$, et al. Down-regulation of vascular endothelial growth factor in renal cell carcinoma cells by glucocorticoids. Mol Cell Endocrinol 2004;226:11-17.

31. Avramis IA, Kwock R, Avramis VI. Taxotere and vincristine inhibit the secretion of the angiogenesis inducing vascular endothelial growth factor (VEGF) by wild-type and drug-resistant human leukemia T-cell lines. Anticancer Res 2001;21:2281-2286.
32. Podar K, Anderson KC. Inhibition of VEGF signaling pathways in multiple myeloma and other malignancies. Cell Cycle 2007;6: 538-542.

33. Eskens FA, Verweij J. The clinical toxicity profile of vascular endothelial growth factor (VEGF) and vascular endothelial growth factor receptor (VEGFR) targeting angiogenesis inhibitors; a review. Eur J Cancer 2006;42:3127-3139. 